\title{
Composition of volatile in micropropagated and field grown aromatic plants from Tuscany Islands
}

\author{
Laura Pistelli ${ }^{\boxplus}$, Cecilia Noccioli², Francesca D’Angiolillo ${ }^{1}$ and Luisa Pistelli2 \\ 1Department of Agriculture, Food and Environment, University of Pisa, Pisa, Italy; 2Department of Pharmacy, University of Pisa, Pisa, Italy
}

\begin{abstract}
Aromatic plant species present in the natural Park of Tuscany Archipelago are used as flavoring agents and spices, as dietary supplements and in cosmetics and aromatherapy. The plants are usually collected from wild stands, inducing a depletion of the natural habitat. Therefore, micropropagation of these aromatic plants can play a role in the protection of the natural ecosystem, can guarantee a massive sustainable production and can provide standardized plant materials for diverse economical purposes. The aim of this study is to compare the volatile organic compounds produced by the wild plants with those from in vitro plantlets using headspace solid phase micro-extraction (HS-SPME) followed by capillary gas-chromatography coupled to mass spectrometry (GC-MS). Typical plants of this natural area selected for this work were Calamintha nepeta L., Crithmum maritimum L., Lavandula angustifolia L., Myrtus communis L., Rosmarinus officinalis L., Salvia officinalis L. and Satureja hortensis L. Different explants were used: microcuttings with vegetative apical parts, axillary buds and internodes. Sterilization percentage, multiplication rate and shoot length, as well as root formation were measured. The volatile aromatic profiles produced from in vitro plantlets were compared with those of the wild plants, in particular for C. maritimum, R. officinalis, S. officinalis and $S$. hortensis. This study indicated that the micropropagation technique can represent a valid alternative to produce massive and sterile plant material characterised by the same aromatic flavour as in the wild grown plants.
\end{abstract}

Key words: VOC, in vitro shoot cultures, aromatic plants, HS-SPME, GC-MS, biodiversity

Received: 03 October, 2012; revised: 15 January, 2013; accepted: 21 February, 2013; available on-line: 25 February, 2013

\section{INTRODUCTION}

The Tuscany Archipelago is the largest marine (and land) Park in Europe, with more than 50000 hectares of sea and about 20000 hectares of land. The Archipelago includes seven main islands as well as some minor islands and rocks (Landi, 1989). The vegetation, influenced by the Mediterranean climate and by the insularity, is characterised by several aromatic species. Typical plants of the Mediterranean that can be observed for example on the Elba Island are adapted to grow near the sea, tolerating salt stress, drought, and rock side soils include Crithmum maritimum L, Rosmarinus officinalis L., Myrtus sp., Spartium sp., Cistus sp., Lavandula sp., Helichrysum spp. and Juniperus spp. (Rinaldi, 2001). These plants constitute an ecological niche for their typical aromatic characteristics and represent an ecological population to exploit and to preserve (Hamilton, 2004; Abraham, 2010). The aromatic plants are usually collected on their natural stands and used as spices in traditional food and cosmetics, in phytotherapy and aromatherapy. This practice harms the natural habitat, so the development of efficient protocols for rapid clonal propagation and the conservation of germplasm of selected species typical of these islands is badly needed. In vitro culture of aromatic and medicinal plants is a useful technique to produce rapidly and in small spaces a large amount of plant material, avoiding the damage and the extinction of natural field grown plants (Debnath et al., 2006; Lucchesini \& Mensuali-Sodi, 2010; Ruffoni et al., 2010). Moreover, micropropagation represents a valid alternative for rapid clonal propagation and an improvement of the production and marketing of the selected plants. The micropropagation protocols are based on regeneration of plants starting from different organs (Lucchesini \& Mensuali-Sodi, 2010). The in vitro culture technique can be also useful for the production of active compounds naturally present in aromatic plants and in particular enhancing the levels of some metabolites (Pistelli et al., 2010).

The plants are able to emit organic volatile compounds (VOC), but the intensity and profile of emission is dependent on the genetic variability and plasticity of phenotypes (Dicke \& Loreto, 2010). VOC are mainly produced by plants for three main reasons: plants-plants interactions, signals for symbiotic organisms, and as insect attractants or repellents. Many VOCs produced by plants are constituents of aromatic essential oils, often used in the food and perfume industries as flavors and/ or fragrances (Maffei et al., 2011). Moreover, it is known that essential oils can have antibacterial, anti-inflammatory and other pharmaceutical uses (Maffei et al., 2011).

Headspace solid phase micro-extraction (HS-SPME) followed by capillary gas-chromatography mass-spectrometry (GC-MS) is currently a widely used technique for the characterization of the composition of plant volatile fraction, due to its easy and fast management (Belliardo et al., 2006).

The aim of the present work was to investigate, for the first time, the chemical composition of the aroma scent emitted by in vitro plantlets (ivP) of several aromatic species, in comparison with the field-grown motherplants (fgP), using HS-SPME. Seven typical species grown in the Tuscany Archipleago were chosen as plant

e-mail: pistelli@agr.unipi.it

Abbreviations: BA, benzyladenine; GC-MS, capillary gas-chromatography mass spectrometry; HS-SPME, headspace solid phase micro-extraction; IAA, indole-3-acetic acid; IBA, indolebutiric acid; MSO, basal Murashige and Skoog medium; NAA, naphtalene acetic acid; PAR, photosynthetically active radiation 
material from different families: Calamintha nepeta (L.) Savi, Lavandula angustifolia Mill., Rosmarinus officinalis L., Salvia officinalis L. and Satureja hortensis L. belonging to Labiatae, Crithmum maritimum L. (Umbelliferae family) and Myrtus communis L. (Myrtaceae family).

\section{MATERIALS AND METHODS}

Plant Materials. Calamintha nepeta L., Crithmum maritimum L., Lavandula angustifolia Mill., Myrtus communis L., Rosmarinus officinalis L., Salvia officinalis L., Satureja hortensis L., representatives of the plant species typical of the Tuscan herbs, were collected in spring 2011 in the Elba Island of the Tuscan Archipelago, and kept in pots in a greenhouse for acclimatization. A voucher specimen was authenticated by S. Maccioni (Università di Pisa, Dipartimento di Biologia) and deposited at the Botanical Garden of the University of Pisa.

Micropropagation. Different types of explants were used: apical portions of $R$. officinalis and $S$. hortensis, internodes with lateral buds for $C$. nepeta, $M$. communis, $L$. angustifolia, and S. officinalis, basal shoots of C. maritimum. The explants from the selected species were differently sterilised. C. nepeta internodes with lateral buds, $10 \mathrm{~mm}$ length, were submerged in $0.05 \%(\mathrm{v} / \mathrm{v})$ Tween-20 ${ }^{\circledR}$ for $20 \mathrm{~min}$, followed by washing with sterile water for $5 \mathrm{~min}$. The explants were then transferred in $50 \%(\mathrm{v} / \mathrm{v})$ commercial hypochlorite for $10 \mathrm{~min}$, and then washed with sterile water for $5 \mathrm{~min}$ (sterilization method called S1) (Grigoriadou \& Maloupa, 2008). Ten-millimetre long internodes of $M$. communis, $L$. officinalis, and $S$. officinalis and basal shoots of $C$. maritimum were treated with detergent $0.05 \%(\mathrm{v} / \mathrm{v})$ Tween- $20^{\circledR}$ for $20 \mathrm{~min}$, then dipped in a $70 \%(\mathrm{v} / \mathrm{v})$ ethanol solution for $1 \mathrm{~min}$ before surface sterilized in $25 \%(\mathrm{v} / \mathrm{v})$ commercial sodium hypochlorite for $10 \mathrm{~min}$, and washed with sterile water for 5 min (S2) (Ruffoni \& Mascarello, 2009). The S3 method, adopted for the apical portions of $\mathrm{R}$. officinalis and $S$. hortensis, comprised a preliminary incubation of the explants in $2 \%(\mathrm{w} / \mathrm{v})$ sucrose and $0.01 \%(\mathrm{v} / \mathrm{v})$ commercial sodium hypochlorite for 15 days in the dark (Ruffoni \& Mascarello, 2009). After washing with water for $5 \mathrm{~min}$, the explants were then sterilized with the S2 treatment. Shoot proliferation was obtained by culturing the sterile explants in different media. The constituents of the basal medium (MS0) were MS macro- and micronutrients, vitamins (Murashige \& Skoog, 1962), 3\% (w/v) sucrose, $0.8 \%(\mathrm{w} / \mathrm{v})$ agar and $0.05 \%(\mathrm{v} / \mathrm{v})$ "Plant preservative mixture" (PPM); the above medium with the addition of $0.5 \mathrm{mg} / \mathrm{L}$ benzyladenine (BA) was called M1. The shoot proliferation medium for $R$. officinalis (M2) contained an extra $0.017 \mathrm{mg} / \mathrm{L}$ indole-3-acetic acid (IAA). The shoot culture multiplication medium for C. maritimum (M3) contained MSO medium with the addition of $0.5 \mathrm{mg} / \mathrm{L}$ $\mathrm{BA}$ and $0.46 \mathrm{mg} / \mathrm{L} \mathrm{NAA}$. The rooting phase was induced in vitro by transferring the shoots to rooting media according to the literature. The following rooting media were utilized: R1, composed of MS0 and $0.2 \mathrm{mg} / \mathrm{L}$ NAA for C. nepeta, R. officinalis and $L$. angustifolia (Andrade et al., 1999); R2 (MS0 with $1 \mathrm{mg} / \mathrm{L} \mathrm{IAA}+1.5 \%$ sucrose) was used for $M$. communis (Scarpa et al., 2000), $\mathrm{R} 3$ (MSO + $0.1 \mathrm{mg} / \mathrm{L} \mathrm{IBA)} \mathrm{for} \mathrm{C.} \mathrm{maritimum} \mathrm{(Grigori-}$ adou \& Maloupa, 2008), and medium R4 (MS0 + 0.5 $\mathrm{mg} / \mathrm{L} \mathrm{IBA}$ ) for $S$. officinalis and $S$. hortensis (Arikat et al., 2004). All media were adjusted to $\mathrm{pH} 5.7$ with $0.1 \mathrm{M}$ $\mathrm{KOH}$ before autoclaving for $20 \mathrm{~min}$ at $120^{\circ} \mathrm{C}$. Cultures were maintained in a growthchamber at $22 \pm 1^{\circ} \mathrm{C}$ under $16 \mathrm{~h}$ light and $8 \mathrm{~h}$ dark photoperiod provided by cool white fluorescent tubes (Philips TLM 40W/33RS) with $80 \mu$ moles $\mathrm{m}^{-2} \mathrm{~s}^{-1}$ PAR. Cultures were monitored regularly for shoot production. Vitality was recorded after one week; after four weeks, the number and length of shoots, and percentage of root formation were recorded and the plantlets were transferred to fresh medium.

SPME analyses. Emitted volatiles were analyzed using a Supelco SPME device coated with polydimethylsiloxane (PDMS, $100 \mu \mathrm{m}$ ) in order to sample the headspace of $10 \mathrm{~g}$ of fresh plant mass comprising either field grown plants or aerial parts without flowers of in vitro plantlets collected at the end of 4-week subculture. Each sample was introduced into a $30 \mathrm{ml}$ glass conical flask and allowed to equilibrate for $30 \mathrm{~min}$. After the equilibration time, the fiber was exposed to the headspace for $15 \mathrm{~min}$ at room temperature; once sampling was finished, the fiber was withdrawn into the needle and transferred to the injector of the GC and GC-MS system, where the fiber was desorbed.

Gas Chromatography-FID. GC analyses were performed using an HP-5890 Series II instrument equipped with HP-WAX and HP-5 capillary columns $(30 \mathrm{~m} \times 0.25$ $\mathrm{mm}, 0.25 \mu \mathrm{m}$ film thickness), working with the following temperature program: $60^{\circ} \mathrm{C}$ for $10 \mathrm{~min}$, rising at $5^{\circ} \mathrm{C}$ / min to $220^{\circ} \mathrm{C}$; splitless injection mode, injector temperature, $250^{\circ} \mathrm{C}$; carrier gas, nitrogen $(2 \mathrm{ml} / \mathrm{min})$; detector, dual FID. The identification of the components was performed for the both columns by comparison of their retention time with those of pure authentic samples and by means of their linear retention indices (IRI) relative to a series of $n$-hydrocarbons. The percentage of the volatile constituents was obtained by FID peak-area normalization.

Gas Chromatography-Mass Spectrometry. GCMS analyses were performed with a Varian CP-3800 gas chromatograph equipped with a DB-5 capillary column $(30 \mathrm{~m} \times 0.25 \mathrm{~mm}$; coating thickness $0.25 \mu \mathrm{m})$ and a Varian Saturn 2000 ion trap mass detector. Analytical conditions: splitless injection mode, injector temperature, $250^{\circ} \mathrm{C}$; oven temperature programmed from 60 ${ }^{\circ} \mathrm{C}$ to $240^{\circ} \mathrm{C}$ at $3^{\circ} \mathrm{C} / \mathrm{min}$; carrier gas, helium at $1 \mathrm{ml} /$ min; injection, $0.2 \mu \mathrm{l}(10 \%$ hexane solution). Identification of the constituents was based on comparison of the retention times with those of authentic samples, comparing their linear retention indices relative to a series of $n$-hydrocarbons, and on computer matching against commercial (NIST 98 and ADAMS) and home-made library mass spectra built up from pure substances, components of known oils and MS literature data (Adams, 1995; Connolly \& Hill, 1991; Jennings \& Shibamoto, 1980; Massada, 1976; Stenhagen et al., 1974; Swigar \& Silverstein, 1981).

\section{RESULTS AND DISCUSSION}

Calamintha nepeta L., Crithmum maritimum L. Lavandula angustifolia Mill., Myrtus communis L., Rosmarinus officinalis L., Salvia officinalis L. and Satureja hortensis L., typical species of the Tuscan Archipelago, were selected for micropropagation with the aim to preserve the environment and to collect the germplasm of typical insular Mediterranean plants. The main type of explants used for the sterilization process were microcutting (vegetative apical portions), in some cases axillary buds or internodes. Various sterilization methods were used to select the best method for each explants. Table 1 shows the sterilization efficiency expressed as the explants'vitality after 7 days of culture. The explants of C. maritimum and $M$. commu- 
Table 1. Vitality percentage, multiplication rate, shoot length, and root formation of micropropagated plants.

Mean values from five independent replicates \pm S.D. are shown. Abbreviations represent respectively: S1, S2 and S3 (sterilization method), M1, M2 and M3 (proliferation media), R1, R2, R3 and R4 (rooting media). For details see the material and methods section.

\begin{tabular}{|c|c|c|c|c|c|}
\hline Species & Explants & Vitality (\%) & Shoot $\mathrm{n} \%$ explant & Shoot height $(\mathrm{cm})$ & Roots (\%) \\
\hline Calaminta nepeta $\mathrm{L}$. & internodal segments & $50(\mathrm{~S} 1)$ & $4 \pm 0.5(\mathrm{M} 1)$ & $2.80 \pm 0.43$ & $100(\mathrm{R} 1)$ \\
\hline Crithmum maritimum L. & basal shoots & $100(S 2)$ & $3 \pm 0.3(\mathrm{M} 3)$ & $2.72 \pm 0.33$ & $40(\mathrm{R3})$ \\
\hline Lavandula angustifolia Mill. & internodal segments & $80(S 2)$ & $3 \pm 0.4(\mathrm{M} 1)$ & $1.84 \pm 0.49$ & $40(\mathrm{R} 1)$ \\
\hline Myrtus communis L. & internodal segments & $100(S 2)$ & $3 \pm 0.5(\mathrm{M} 1)$ & $3.27 \pm 0.51$ & $79(\mathrm{R} 2)$ \\
\hline Rosmarinus officinalis L. & apical portions & $80(\mathrm{S3})$ & $1 \pm 0.1(\mathrm{M} 2)$ & $1.50 \pm 0.50$ & $0(\mathrm{R} 1)$ \\
\hline Salvia officinalis L. & internodal segments & $10(\mathrm{~S} 2)$ & $2 \pm 0.3(\mathrm{M} 1)$ & $4.29 \pm 0.41$ & $100(\mathrm{R} 4)$ \\
\hline Satureja hortensis L. & apical portions & $80(S 3)$ & $1 \pm 0.2(\mathrm{M} 1)$ & $2.90 \pm 1.17$ & 5 (R4) \\
\hline
\end{tabular}

nis gave the best results of sterilization, reaching $100 \%$ vitality, not contaminated, as indicated in the literature (Grigoriadou \& Maloupa, 2008; Ruffoni \& Mascarello, 2009). A good percentage of success, $80 \%$, was also observed for apical portions of $R$. officinalis and $S$. hortensis as well as for internodes of $L$. angustifolia. The method S3 included a pre-treatment with sucrose for 15 days, already successfully demonstrated in other Mediterranean explants (Ruffoni \& Mascarello, 2009). A lower percentage of vitality was observed for explants of $C$. nepeta $(50 \%)$ and for $S$. officinalis, as known from the literature (Avato et al., 2005). The best explants were propagated using different media, chosen for simplicity of use, and subcultured every 4 weeks. The multiplication of shoots and their length were evaluated at the end of the cycle of subculture (Table 1). M1 medium, with addition of only BA as growth regulator, exhibited good results for $M$. communis, $C$. nepeta, $L$. angustifolia and $S$. officinalis; this confirms that proliferation can be achieved also with a minimum addition of growth regulators, as often reported in the literature for some species (Scarpa et al., 2000; Avato et al., 2005; Ruffoni \& Mascarello, 2009). However, for C. maritimum the highest number of shoots was obtained adding both auxin (NAA) and cytokinin (BA) to the MS0 medium (Grigoriadou \& Maloupa, 2008). S. hortensis produced modest results of propagation when apical portions were used as explants, confirming the literature data for S. obovata (Arrebola, 1997). R. officina-

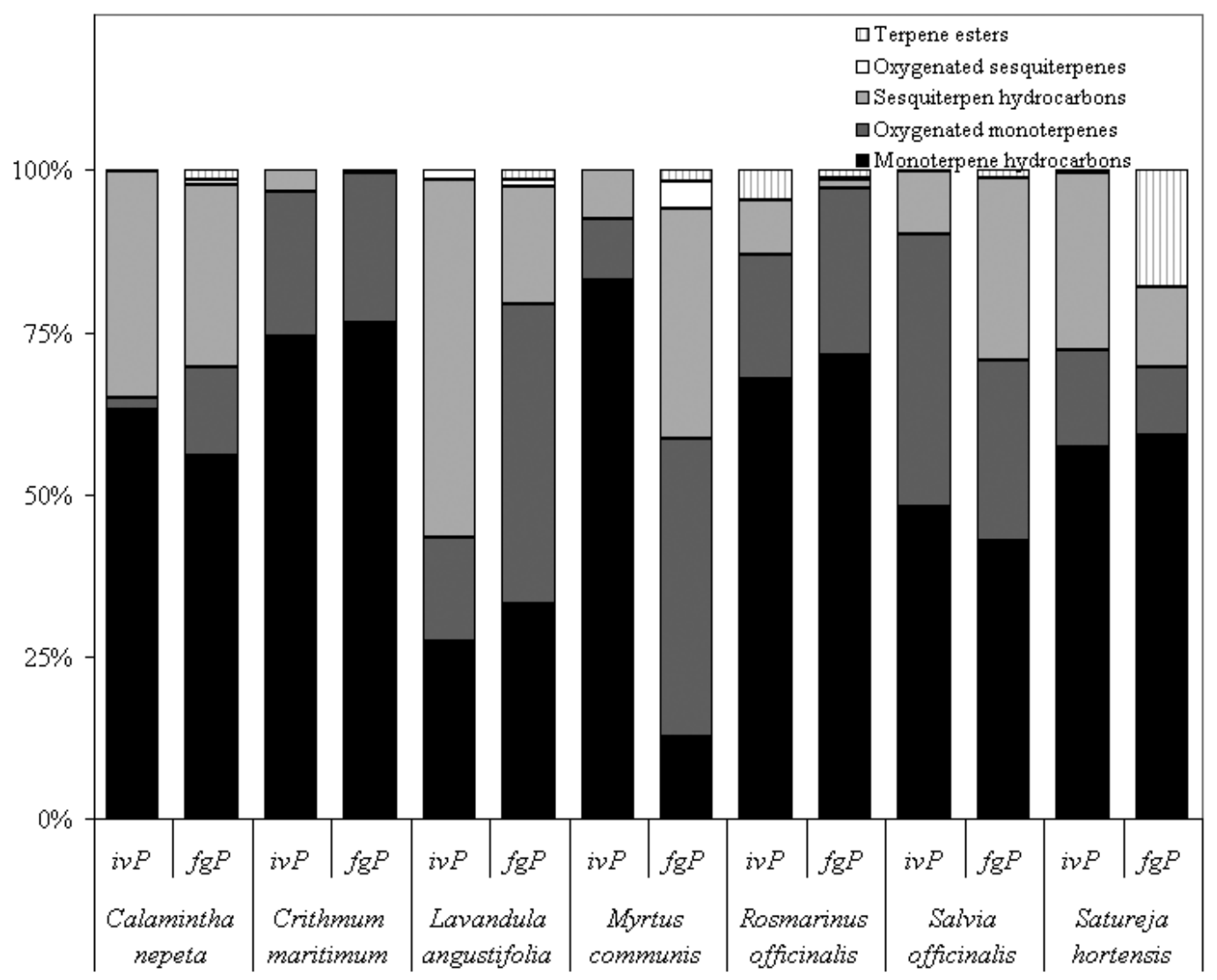

Figure 1. Terpene composition of the headspace of plants grown in the field (fgP) and in vitro (ivP). 
Table 2. Main constituents identified in HS-SPME of examinated species

\begin{tabular}{|c|c|c|c|c|c|c|c|c|c|c|c|c|c|c|c|c|}
\hline \multirow{2}{*}{ Compound } & & \multirow[b]{2}{*}{$|R|$} & \multicolumn{2}{|c|}{ C. nepeta } & \multicolumn{2}{|c|}{ C. maritimum } & \multicolumn{2}{|c|}{$\begin{array}{l}\text { L. angusti- } \\
\text { folia }\end{array}$} & \multicolumn{2}{|c|}{$\begin{array}{l}\text { M. commu- } \\
\text { nis }\end{array}$} & \multicolumn{2}{|c|}{ R. officinalis } & \multicolumn{2}{|c|}{ S. officinalis } & \multicolumn{2}{|c|}{ S. hortensis } \\
\hline & & & $\begin{array}{l}\% \\
\text { ivP }\end{array}$ & $\begin{array}{l}\% \\
f g P\end{array}$ & $\%$ ivP & $\begin{array}{l}\% \\
f g P\end{array}$ & $\begin{array}{l}\% \\
\text { ivP }\end{array}$ & $\begin{array}{l}\% \\
f g P\end{array}$ & $\begin{array}{l}\% \\
\text { ivP }\end{array}$ & $\begin{array}{l}\% \\
f g P\end{array}$ & $\begin{array}{l}\% \\
\text { ivP }\end{array}$ & $\begin{array}{l}\% \\
\mathrm{fgP}\end{array}$ & $\begin{array}{l}\% \\
\text { ivP }\end{array}$ & $\begin{array}{l}\% \\
\mathrm{fgP}\end{array}$ & $\begin{array}{l}\% \\
\text { ivP }\end{array}$ & $\begin{array}{l}\% \\
f g P\end{array}$ \\
\hline a-thujene & $\mathrm{MH}$ & 932 & & & 1.2 & 0.3 & & 0.4 & & & 0.3 & 0.9 & 0.3 & 0.8 & 1.5 & 1.4 \\
\hline tricyclene & $\mathrm{MH}$ & 938 & & & & & & & 65.0 & & & & & & & \\
\hline a-pinene & $\mathrm{MH}$ & 940 & 1.8 & 6.3 & 10.3 & 11.9 & 1.4 & 4.6 & 4.2 & 4.8 & 24.7 & 34.4 & 14.6 & 5.3 & 1.5 & 3.0 \\
\hline camphene & $\mathrm{MH}$ & 955 & 1.8 & & 0.4 & & 0.6 & 1.6 & & & 13.4 & 19.7 & 8.5 & 1.9 & 0.1 & 3.3 \\
\hline$\beta$-thujene & $\mathrm{MH}$ & 971 & & & 1.0 & & & & & & & & & & & \\
\hline sabinene & $\mathrm{MH}$ & 978 & 1.2 & 1.4 & 15.0 & 1.5 & 0.5 & 0.4 & & & & & & & 0.2 & 0.2 \\
\hline$\beta$-pinene & $\mathrm{MH}$ & 981 & 42.5 & 8.4 & & & 2.4 & 2.3 & & & 9.6 & 10.5 & 19.4 & 10.1 & 1.0 & 1.8 \\
\hline myrcene & $\mathrm{MH}$ & 993 & & 2.1 & 1.7 & 0.9 & 1.6 & 1.9 & & & 10.8 & 3.2 & 1.4 & 11.0 & 1.0 & 1.7 \\
\hline a-phellandrene & $\mathrm{MH}$ & 1006 & & & & & 0.7 & 1.4 & & & 0.1 & & & & 0.3 & \\
\hline$\delta$-3-carene & $\mathrm{MH}$ & 1012 & & 0.3 & & & 6.9 & 11.3 & & & & & & & 0.1 & \\
\hline a-terpinene & $\mathrm{MH}$ & 1019 & & & 0.3 & 0.4 & & & & & 0.4 & & 0.1 & 0.3 & 3.4 & 2.5 \\
\hline o-cymene & $\mathrm{MH}$ & 1026 & & & & & & & & & 0.5 & & 0.1 & 1.1 & & \\
\hline$p$-cymene & $\mathrm{MH}$ & 1028 & & & 9.6 & 10.1 & 1.0 & 0.6 & & 2.1 & & 0.6 & & & 15.6 & 30.3 \\
\hline$\beta$-phellandrene & $\mathrm{MH}$ & 1031 & & & & & 6.2 & & & & & & & & & \\
\hline limonene & $\mathrm{MH}$ & 1032 & 15.0 & 34.8 & & & & & & 2.5 & 4.0 & & 2.3 & 4.3 & 1.2 & \\
\hline 1,8-cineol & $\mathrm{MH}$ & 1036 & & & & & 11.9 & 22.2 & & 32.5 & 10.7 & 19.2 & 7.2 & 5.8 & & 1.2 \\
\hline$(Z)$ - $\beta$-ocimene & $\mathrm{MH}$ & 1042 & & & 0.6 & & & 5.3 & & & & & & 4.4 & & \\
\hline$(E)$ - $\beta$-ocimene & $\mathrm{MH}$ & 1053 & & & & & & 0.4 & & & & & & 0.4 & & \\
\hline $\mathrm{Y}$-terpinene & $\mathrm{MH}$ & 1062 & & 0.4 & 30.9 & 49.3 & 0.4 & 0.1 & 2.9 & & 1.5 & 0.2 & 0.4 & 1.1 & 29.0 & 11.7 \\
\hline$p$-mentha-3,8-diene & $\mathrm{MH}$ & 1072 & & & & & & & 2.0 & & & & & & & \\
\hline cis-sabinene hydrate & $\mathrm{MH}$ & 1072 & & & & & & 0.5 & & & & 0.2 & & & & \\
\hline$p$-cymenene & $\mathrm{MH}$ & 1080 & & & & & & & & & & & & & 0.4 & 0.8 \\
\hline isoterpinolene & $\mathrm{MH}$ & 1086 & & & & & 0.5 & 0.8 & & & & & & & & \\
\hline terpinolene & $\mathrm{MH}$ & 1090 & 0.1 & 0.3 & 1.3 & & 1.4 & 0.7 & & & 1.7 & 0.3 & 0.9 & 0.6 & & \\
\hline linalool & MO & 1102 & & & & & & & & & & 0.5 & & 0.4 & & 0.5 \\
\hline nonanal & ALD & 1104 & & & & & & & & 1.7 & & & & & & \\
\hline a-thujone & MO & 1109 & & & & & & & & & & & 20.7 & 9.2 & & 0.4 \\
\hline$\beta$-thujone & MO & 1120 & & & & & & & & & & & 4.5 & 3.1 & & \\
\hline cis-limonene oxide & MO & 1137 & 0.2 & 0.4 & & & & & & & & & & & & \\
\hline neo-alloocimene & $\mathrm{MH}$ & 1142 & & & & & & & & & & & & 0.6 & & \\
\hline camphor & $\mathrm{MO}$ & 1148 & & & & & & 21.5 & & & 7.9 & 1.4 & 8.8 & 6.9 & & 0.3 \\
\hline menthone & MO & 1154 & 0.5 & 0.4 & & & & & & & & & & & & \\
\hline menthofuran & $\mathrm{MO}$ & 1164 & 0.5 & 0.7 & & & & & & & & & & & & \\
\hline trans-pinocamphone & MO & 1165 & & & & & & & & & & & 0.4 & & & \\
\hline borneol & MO & 1169 & & & & & 1.8 & 1.1 & & 0.9 & & 3.5 & & 1.4 & & 2.0 \\
\hline cis-pinocamphone & $\mathrm{MO}$ & 1177 & & & & & & & & & & 0.3 & 0.2 & 0.3 & & \\
\hline myrtenal & $\mathrm{MO}$ & 1184 & & 1.8 & & & & & & & & & & & & \\
\hline a-terpineol & $\mathrm{MO}$ & 1192 & & & & & 0.2 & 0.2 & & 0.9 & & 0.2 & & & & \\
\hline 1-dodecene & ALK & 1192 & & & & & 1.8 & & & & & & & & & \\
\hline decanal & ALD & 1206 & & & & & & & & 1.4 & & & & & & \\
\hline verbenone & MO & 1214 & & & & & & 0.2 & & & 0.3 & 0.1 & & & & \\
\hline methyl thymol & $\mathrm{MO}$ & 1235 & & & 0.4 & 0.3 & & & 7.2 & & & & & & 0.2 & 11.9 \\
\hline pulegone & MO & 1237 & & 0.5 & & & & & & & & & & & & \\
\hline methyl carvacrol & $\mathrm{MO}$ & 1244 & & & 21.1 & 22.0 & & & 1.2 & & & & & & 0.3 & 4.1 \\
\hline piperitone oxide & MO & 1257 & & 4.9 & & & & & & & & & & & & \\
\hline
\end{tabular}




\begin{tabular}{|c|c|c|c|c|c|c|c|c|c|c|c|c|c|c|c|c|}
\hline bornyl acetate & TE & 1287 & & & & & & 1.2 & & & 4.5 & 1.2 & 0.2 & 0.8 & & 1.4 \\
\hline thymol & MO & 1293 & & & & & & & & & & & & & & 5.5 \\
\hline lavandulyl acetate & TE & 1289 & & & & & & 0.2 & & & & & & & & \\
\hline$n$-tridecane & ALK & 1300 & & & & & 2.0 & & & & & & & & & \\
\hline carvacrol & MO & 1301 & 0.5 & & & & & & & & & & & & 14.4 & \\
\hline myrtenyl acetate & $\mathrm{TE}$ & 1327 & & & & & & & & 1.3 & & & & & & \\
\hline$\delta$-elemene & $\mathrm{SH}$ & 1340 & & & & & & & & & & & & 0.4 & & \\
\hline piperitenone & MO & 1342 & & 0.4 & & & & & & & & & & & & \\
\hline piperitenone oxide & $\mathrm{MO}$ & 1363 & & 4.1 & & & & & & & & & & & & \\
\hline cyclosativene & $\mathrm{SH}$ & 1371 & & & & & & & & & & & & 0.7 & & \\
\hline a-copaene & $\mathrm{SH}$ & 1376 & 0.1 & 0.7 & & & & & & & & & & 0.6 & & \\
\hline$\beta$-bourbonene & $\mathrm{SH}$ & 1383 & & 1.1 & & & & & & & & & & & & \\
\hline$\beta$-cubebene & $\mathrm{SH}$ & 1390 & 0.9 & 0.3 & & & & & & & & & & & & \\
\hline 7-epi-sesquithujene & $\mathrm{SH}$ & 1391 & & & & & 0.9 & & & & & & & & & \\
\hline$\beta$-elemene & $\mathrm{SH}$ & 1392 & & 0.5 & & & & 0.3 & & 6.9 & & & & & & \\
\hline n-tetradecane & ALK & 1400 & & & & & 0.5 & & & & & & & & & \\
\hline isocaryophyllene & $\mathrm{SH}$ & 1406 & & & & & 0.4 & & & & & & & & 0.2 & \\
\hline a-cedrene & $\mathrm{SH}$ & 1409 & 0.6 & & & & & & & & & & & & & \\
\hline a-gurjunene & $\mathrm{SH}$ & 1410 & & & & & 2.1 & 3.0 & & & & & & 0.3 & & \\
\hline caryophyllene & $\mathrm{SH}$ & 1418 & 2.7 & 10.3 & & 0.1 & 19.3 & 6.5 & 6.8 & 8.1 & 5.0 & 1.1 & 1.5 & 3.3 & 20.0 & 5.0 \\
\hline$\beta$-gurjunene & $\mathrm{SH}$ & 1428 & 1.8 & 0.5 & & & 0.3 & & & & & & & 0.2 & & \\
\hline trans-a-bergamotene & $\mathrm{SH}$ & 1437 & & 0.5 & 0.6 & & 3.3 & & & & & & & & 0.1 & \\
\hline cis-muurola-3,5-diene & $\mathrm{SH}$ & 1448 & 13.9 & & & & 0.9 & 0.7 & & & & & & & & \\
\hline aromadendrene & $\mathrm{SH}$ & 1445 & & 0.4 & & & 0.3 & & & & & & & 2.0 & & \\
\hline a-himachalene & $\mathrm{SH}$ & 1453 & 0.2 & 0.4 & & & & & & & & & & & & \\
\hline a-humulene & $\mathrm{SH}$ & 1456 & & 0.7 & & & 0.8 & 0.2 & & 3.0 & 3.5 & 0.1 & 8.1 & 11.3 & 1.1 & 0.6 \\
\hline$(E)$ - $\beta$-farnesene & $\mathrm{SH}$ & 1460 & & & & & 1.7 & & & & & & & & & \\
\hline $\begin{array}{l}\text { cis-muurola-4(14),5- } \\
\text {-diene }\end{array}$ & $\mathrm{SH}$ & 1463 & 9.1 & & & & 1.1 & 0.7 & & & & & & & & \\
\hline $\mathrm{\gamma}$-muurolene & $\mathrm{SH}$ & 1477 & & & & & & & & & & & & 1.4 & & \\
\hline germacrene D & $\mathrm{SH}$ & 1481 & 2.0 & 10.7 & 0.7 & 0.3 & 8.5 & 2.1 & & & & & & 2.0 & & 2.2 \\
\hline$\beta$-selinene & $\mathrm{SH}$ & 1485 & 1.3 & & & & 0.4 & & & 3.8 & & & & & & \\
\hline a-zingiberene & $\mathrm{SH}$ & 1495 & & & 0.6 & & & & & & & & & & & \\
\hline bicyclogermacrene & $\mathrm{SH}$ & 1495 & 0.1 & & & & 1.1 & 0.1 & & & & & & 3.2 & 1.0 & 0.5 \\
\hline a-selinene & $\mathrm{SH}$ & 1497 & & & & & & & & 4.7 & & & & & & \\
\hline$n$-pentadecane & ALK & 1500 & & & & & 1.9 & & & & & & & & & \\
\hline$(E, E)$-a-farnesene & $\mathrm{SH}$ & 1508 & & & & & 0.8 & & & & & & & & & \\
\hline$\beta$-bisabolene & $\mathrm{SH}$ & 1509 & 0.1 & 0.5 & 0.3 & & & & & & & & & & 4.0 & 3.5 \\
\hline trans- $\gamma$-cadinene & $\mathrm{SH}$ & 1513 & 0.2 & & & & 3.8 & 3.8 & & & & & & 0.4 & & \\
\hline trans-calamenene & $\mathrm{SH}$ & 1522 & 1.1 & & & & & 0.1 & & & & & & & & \\
\hline$\delta$-cadinene & $\mathrm{SH}$ & 1523 & & 0.3 & & & 1.5 & & & & & & & 1.0 & & \\
\hline$\beta$-sesquiphellandrene & $\mathrm{SH}$ & 1524 & & & 0.5 & & & & & & & & & & & \\
\hline caryophyllene oxide & SO & 1582 & & & & & & 0.5 & & 3.2 & & 0.3 & & & & \\
\hline 1,10-di-epi-cubenol & SO & 1614 & 0.2 & 0.4 & & & 0.4 & & & & & & & & & \\
\hline germacrene B & $\mathrm{SH}$ & 1556 & & & 0.4 & 0.1 & & & & & & & & & & \\
\hline diilapiol & PP & 1623 & & & 0.6 & 2.3 & & & 3.4 & & & & & & & \\
\hline epi-a-cadinol & SO & 1642 & & & & & 0.9 & 0.5 & & & & & & & & \\
\hline pentyl decanoate & $\mathrm{AE}$ & & & & & & & & & 13.6 & & & & & & \\
\hline a-bisabolol & SO & 1684 & & 0.4 & & & & & & & & & & & & \\
\hline
\end{tabular}




\begin{tabular}{|c|c|c|c|c|c|c|c|c|c|c|c|c|c|c|c|c|}
\hline n-heptadecane & ALK & 1700 & & & & & 0.6 & & & 0.9 & & & & & & \\
\hline n-octadecane & ALK & 1800 & & & & & & & & 0.9 & & & & & & \\
\hline $\begin{array}{l}\text { Monoterpene hydro- } \\
\text { carbons }\end{array}$ & $\mathrm{MH}$ & & 62.4 & 54.0 & 72.3 & 74.4 & 23.6 & 32.8 & 74.1 & 9.4 & 67.2 & 70.0 & 48.0 & 41.9 & 55.3 & 56.7 \\
\hline $\begin{array}{l}\text { Oxygenated mono- } \\
\text { terpenes }\end{array}$ & MO & & 1.7 & 13.2 & 21.5 & 22.3 & 13.9 & 45.5 & 8.4 & 34.3 & 18.9 & 25.4 & 41.8 & 27.3 & 14.4 & 9.9 \\
\hline $\begin{array}{l}\text { Sesquiterpen hydro- } \\
\text { carbons }\end{array}$ & $\mathrm{SH}$ & & 34.6 & 26.9 & 3.3 & 0.5 & 47.6 & 18 & 6.8 & 26.5 & 8.5 & 1.2 & 9.6 & 27.7 & 26.4 & 11.8 \\
\hline $\begin{array}{l}\text { Oxygenated sesqu- } \\
\text { iterpenes }\end{array}$ & SO & & 0.2 & 0.8 & & & 1.3 & 1.0 & & 3.2 & & 0.3 & & & & \\
\hline Terpene esters & TE & & & 1.5 & & & & 1.5 & & 1.3 & 4.5 & 1.2 & 0.2 & 1.2 & 0.5 & 17.4 \\
\hline Phenylpropanoids & PP & & & & 0.6 & 2.3 & & & 3.4 & & & & & & & \\
\hline Alkanes & ALK & & & & & & 7.4 & & & 1.8 & & & & & & 0.2 \\
\hline Adeydes & ALD & & & & & & 0.2 & & & 3.1 & & & & & & 0.5 \\
\hline Acids/esters & $\mathrm{AE}$ & & & 0.3 & & & & & & 13.6 & & & & & & \\
\hline Total & & & 98.4 & 94.9 & 97.5 & 99.5 & 92.8 & 97.4 & 92.7 & 93.2 & 98.9 & 97.9 & 99.6 & 96.6 & 96.6 & 95.8 \\
\hline
\end{tabular}

$\mid R I$, Linear retention index. The components are listed in order of their elution on the DB-5 column. IvP, in vitro plants; fgP, field-grown plants. MH, monoterpene hydrocarbons; $\mathrm{MO}$, oxygenated monoterpenes; $\mathrm{SH}$, sesquiterpene hydrocarbons; $\mathrm{SO}$, oxygenated sesquiterpenes; $\mathrm{TE}$, terpene esters; ALD, aldehydes; ALK, alkanes/alkenes; PP, phenyl propanoids; $A E$, esters. Compounds present at less than $0.1 \%$ were excluded from the table and from analysis.

lis exhibited the lowest number of shoot proliferation even in a specific medium (M2), and no root formation was observed (R1 medium), confirming the refractivity of this species to proliferation (Misra \& Chaturvedi, 1984). The shoot length is also shown in Table 1 , and the varied growth ability of the explants confirmed the data already known from the literature. Notably, we devised a good method for the in vitro propagation of $C$. nepeta, not considered for micropropagation until now (Misra \& Chaturvedi, 1984, Arrebola, 1997; Avato et al., 2005; Grigoriadou \& Maloupa, 2008). Root formation was then induced in order to produce aseptically whole plants to be easily transferred in diverse environments. Best results were obtained with C. nepeta and S. officinalis, whereas the percentage of produced roots was lower for other species.

The volatiles emitted from the analysed species and identified by GC-MS are reported in Table 2. In total, 71 and 78 compounds were identified in micropropagated (ivP) and field-grown (fgP) plants. The headspace analyses of these two growth conditions accounted for $92.7-99.6 \%$ and $93.2-99.5 \%$ of the total compositions, respectively. The volatile fractions were characterized mainly by hydrocarbons and oxygenated monoterpenes together with sesquiterpene hydrocarbons (Fig. 1). The SPME analysis of the two samples of C. nepeta showed a similar composition in monoterpenes (64.1 and $67.2 \%$ in ivP and foP, respectively) and sesquiterpenes (34.1 and $26.9 \%$ in ivP and fgP, respectively), even if fgP exhibited higher percentages of oxygenated monoterpenes $(13.2 \%)$ than ivP $(1.7 \%)$. In fgP typical compounds of Calamintha spp. were detected, as limonene $(34.8 \%)$, piperitone oxide and piperitenone oxide (4.9 and $4.1 \%$, respectively), together with low amounts of pulegone and menthone, ( 0.5 and $0.4 \%$, respectively), in accordance with literature data (De Pooter et al., 1986). In contrast, ivP showed the presence of cis-muurola 3,5-diene (13.9\%) and cis-muurola-4(14),5-diene (9.1\%), not detected in fgP, and a high percentage of $\beta$-pinene $(42.5 \%$, against $8.4 \%$ in $\mathrm{fgP})$. The headspaces of C. maritimum samples showed a similar composition, characterised by monoterpene hydrocarbons $(72.3 \%$ in ivP and $74.4 \%$ in fgP) and oxygenated monoterpenes $(21.5 \%$ in ivP and $22.3 \%$ in foP), differing only by the percentages of sabinene $(15.0 \%$ in ivP and $1.5 \%$ in fgP) and $\gamma$-terpinene $(30.9 \%$ in ivP and 49.3 in foP). The identified constituents are in agreement also with the composition of the essential oils reported in the literature (Özcan et al., 2006), even if many reports on the essential oil composition of sea fennel grown in different parts of Mediterranean area showed differences in chemical constituents, suggesting different chemotypes of this species (Kulisic-Bilusic et al., 2010). The two L. angustifolia samples showed qualitative differences in the composition of the volatiles emitted: the ivP headspace was characterised especially by sesquiterpene hydrocarbons $(47.2 \%)$, while fgP exhibited high percentages of oxygenated and hydrocarbon monoterpenes $(45.2 \%$ and $32.3 \%$, respectively). 1,8-cineol, the compound that gives the particular flavour of the lavender fields, was detected in both samples $(11.9 \%$ in ivP and $22.2 \%$ in fgP), while camphor was identified only in the headspace of fgP $(21.5 \%)$. These results represent new data regarding the head space composition of the vegetative aerial part of $L$. angustifolia, since earlier reports focused on the flower aroma and/or the reproductive aerial parts (Kim et al., 2002, Da Porto \& Decorti 2008). SPME analyses of micropropagated $L$. viridis plants and field-grown motherplant exhibited a different composition of the emitted volatiles (Gonçalves et al., 2008). The headspace analysis obtained from field-grown $M$. communis showed a similar composition with the literature data (Flamini et al., 2004), with the presence of 1,8 -cineol $(32.5 \%), \alpha$-pinene $(4.8 \%)$, limonene $(2.5 \%)$ and mirtenyl acetate $(1.3 \%)$. In contrast, in the SPME analysis of ivP, tricyclene was revealed as the main constituent $(65.0 \%)$, together with methyl thymol $(7.2 \%)$, showing a very different composition from the field-grown plant. The SPME analysis of $\mathrm{R}$. officinalis samples showed the presence of similar percentages of monoterpene hydrocarbons $(67.0 \%$ in ivP and $70.0 \%$ in $\mathrm{fgP})$, but quantitative differences in the percentages of oxygenated monoterpenes $(18.9 \%$ in ivP and $25.2 \%$ in $\mathrm{fgP})$ and of sesquiterpene hydrocarbons (8.5\% in ivP and $1.2 \%$ in fgP). $\alpha$-pinene $(24.7 \%$ and $34.4 \%$ in ivP and fgP, respectively). 1,8-cineol (10.7\% in ivP and $19.2 \%$ in fgP) and camphor (7.9\% in ivP and $1.4 \%$ in fgP) represent the most important constituents of the essential 
oil and aroma of R. officinalis (Katerinopoulos et al., 2005; Zawirska-Wojtasiak \& Wasowicz, 2009). The main compounds identified in the headspace of both samples of S. officinalis were $\alpha$-thujone $(20.7 \%$ and $9.2 \%$ in ivP and fgP, respectively), $\beta$-pinene $(19.4 \%$ in ivP and $10.1 \%$ in $\mathrm{fgP})$ and $\alpha$-pinene $(14.6 \%$ and $5.3 \%$ in ivP and fgP, respectively), together with camphor $(8.8 \%$ in ivP and $6.9 \%$ in fgP) and $\alpha$-humulene (8.1\% in ivP and $11.3 \%$ in $\mathrm{fgP}$ ), in agreement with the quali-quantitative composition reported for $S$. officinalis essential oil (Longaray Delamare et al., 2007; Santos-Gomes et al., 2001). Despite the presence of compounds characteristic for $S$. officinalis in both analysed samples, the micropropagated plants showed higher content of oxygenated monoterpenes $(41.8 \%)$ then the field-grown plants $(27.1 \%)$, while the sesquiterpene hydrocarbons were present especially in the field-grown samples $(26.8 \%$ and $9.6 \%$ in ivP and fgP, respectively). The headspace of the $S$. hortensis two samples was characterised mainly by $\gamma$-terpinene $(29.0 \%$ in ivP and $11.7 \%$ in $\mathrm{fgP}), p$-cymene $(15.6 \%$ in ivP and $30.3 \%$ in fgP), carvacrol (14.4\%, identified only in ivP) and methylthymol and thymol (11.9 and 5.5\%, respectively, the both detected only in the field-grown plants), characteristic constituents of the essential oil from aerial parts. $\gamma$-terpinene and $p$-cymene are biogenetic precursors (via enzymatic hydroxylation) of the phenolic terpenes thymol and carvacrol, so there is a correlation between these compounds and their percentages are affected by harvesting time and location (Azaz et al., 2005; Sefidkon et al., 2006; Güllüce et al., 2003; Abu-Lafi et al., 2008).

Many VOCs produced by officinal and medicinal plants are commercialized as flavors and/or fragrances, and their use in the food and perfume industries has a long tradition. This work showed that the headspace composition of micropropagated plantlets of C. maritimum, R. officinalis, $S$. officinalis and $S$. hortensis is very similar to the corresponding field-grown plants, containing the same main constituents, although further investigations are requested for C. nepeta, $L$. angustifolia and $M$. communis in order to obtain in vitro plant material with aromatic profile more similar to the wild plants. These results indicated that micropropagation can represent a valid alternative to produce rapidly large amounts of plant material characterised by the same aromatic flavor as the wild grown plants. Other advantages of this technique are the protection of the natural ecosystem avoiding the damage of endemic plants. The emission of VOC by the micropropagated plants can represent a good market actractant for baby plants. Customers may use them as spices or as steril ornamental plants to be transported everywhere.

\section{Acknowledgments}

This work was supported by the Interreg IT-FR Marittimo Project "PYRGI, CUP B51H10000000006, Strategia d'impresa in settori di nicchia per l'economia agroindustriale del mediterraneo".

\section{REFERENCES}

Abraham Z (2011) Collection and conservation of medicinal and aromatic plants resources. OAJMAP 1: 40-43.

Abu-Lafi S, Odeh I, Dewik H, Qabajah M, Hanus LO, Dembitsky VM (2008) Thymol and carvacrol production from leaves of wild palestinian Majorana syriaca. Bioresour Technol. 99: 3914-3918.

Adams RP (1995) In Identification of essential oil components by gas chromatography-mass spectroscopy. Allured, Carol Stream, Illinois.

Andrade LB, Echeverrigaray S, Fracaro F, Pauletti GF, Rota L (1999) The effect of growth regulators on shoot propagation and rooting of common lavender (Lavandula vera DC). Plant Cell Tissue Org Cult 56: $79-83$.
Arikat NA, Jawad M, Karam NS, Shibli RA (2004) Micropropagation and accumulation of essential oils in wild sage (Salvia fruticosa Mill.) Sci Hortic 100: 193-202.

Arrebola ML, Socorro O, Barceló-Muñoz A, Simón-Pérez E, PliegoAlfaro F (1997) Micropropagation of Satureja obovata Lag. Hort Sci 32: $1278-1280$.

Avato P, Morone Fortunato I, Ruta C, D'Elia R (2005) Glandular hair's and essential oils in micropropagated plants of Salvia officinalis L. Plant Sci 169: 29-36.

Azaz AD, Kürkcüoglu M, Satil F, Can Baser KH, Tümen G (2005) In vitro antimicrobial activity and chemical composition of some Satureja essential oils. Flavour Fragr J 20: 587-591.

Belliardo F, Bicchi C, Cordero C, Liberto E, Rubiolo P, Sgorbini (2006) Headspace solid-phase microextraction in the analysis of the volatile fraction of aromatic and medicinal plants. J Chrom Sci 44: 416-429.

Connolly JD, Hill RA (1991) In Dictionary of terpenoids. Chapman \& Hall, London.

Da Porto C, Decorti D (2008) Analysis of the volatile compounds of flowers and essential oils from Lavandula angustifolia cultivated in northeastern italy by headspace solid-phase microextraction coupled to gas chromatography-mass spectrometry. Planta Med 74: 182-187.

De Pooter HL, De Buyck LF, Schamp NM (1986) The volatiles of Calamintha nepeta subsp. glandulosa. Phytochemistry 25: 691-694.

Debnath M, Malik CP, Bisen PS (2006) Micropropagation: a tool for the production of high quality plant-based medicines. Curr Pharm Biotechnol 7: 33-49.

Dicke M, Loreto F (2010) Induced plant volatiles: from genes to climate change. Trends Plant Sci 15: 115-117.

Flamini G, Cioni PL, Morelli I, Maccioni S, Baldini R (2004) Phytochemical typologies in some populations of Myrtus communis L. on Caprione Promontory (East Liguria, Italy). Food Chem 85: 599-604.

Gonçalves S, Serra H, Nogueira JMF, Almeida R, Custódio L, Romano A (2008) Headspace-SPME of in vitro shoot-cultures and micropropagated plants of Lavandula viridis. Biol Plantarum 52: 133-136.

Grigoriadou K, Maloupa E (2008) Micropropagation and salt tolerance of in vitro grown Crithmum maritimum L. Plant Cell Tiss Org Cult 94: 209-217.

Güllüce M, Sökmen M, Daferera D, Agar G, Özkan H, Kartal N, Polissiou M, Sökmen A, Sahin F (2003) In vitro antibacterial, antifungal, and antioxidant activities of the essential oil and methanol extracts of herbal parts and callus cultures of Satureja hortensis L. J Agric Food Chem 51: 3958-3965.

Hamilton AC (2004) Medicinal plants, conservation and livelihoods. Biodivers Conserv 13: 1477-1517.

Jennings W, Shibamoto T (1980) In Qualitative analysis of flavor and fragrance volatiles by glass capillary gas chromatography. Academic Press, New York.

Katerinopoulos HE, Pagona G, Afratis A, Stratigakis N, Roditakis N (2005) Composition and insect attracting activity of the essential oil of Rosmarinus officinalis. J Chem Ecol 31: 111-122.

Kim NS, Lee DD (2002) Comparison of different extraction method for the analysis of fragrance from Lavandula species by gas chromatographymass spectrometry. J Chromatogr A 982: 31-47.

Kulisic-Bilusic T, Blazevic I, Dejanovic B, Milos M, Pifat G (2010) Evaluation of the antioxidant activity of essential oils from caper (Capparis spinosa) and sea fennel (Crithmum maritimum) by different methods. J Food Biochem 34: 286-302.

Landi S (1989) In Flora e ambiente dell'isola d'Elba. Editrice Azzurra, Cavalese (Tn).

Longaray Delamare AP, Moschen-Pistorello IT, Artico L, Atti-Serafini L, Echeverrigaray S (2007) Antibacterial activity of the essential oils of Salvia officinalis L. and Salvia triloba L. cultivated in South Brazil. Food Chem 100: 603-608.

Lucchesini M, Mensuali-Sodi A (2010) Plant tissue culture-An opportunity for the production of nutraceuticals. Adv Exp Med Biol 698: 185-202.

Maffei M, Gertsch J, Appendino G (2011) Plant volatiles: production, function and pharmacology. Nat Prod Rep 28: 1359-1380.

Massada Y (1976) In Analysis of essential oils by gas chromatography and mass spectrometry. Wiley \& Sons, New York.

Misra P, Chaturvedi HC (1984) Micropropagation of Rosmarinus officinalis L. Plant Cell Tiss Org Cult 3: 163-168.

Murashige T, Skoog F (1962) A revised medium for rapid growth and bioassays with tobacco tissue cultures. Physiol Plant 15: 473-493.

Özcan MM, Pedro LG, Figueiredo AC, Barroso JG (2006) Constituents of the essential oil of sea fennel (Crithmum maritimum L.) growing wild in Turkey. J Med Food 9: 128-130.

Pistelli L, Giovannini A, Ruffoni B, Bertoli A, Pistelli L. (2010) Hairy root cultures for secondary metabolites production. Adv Exp Med Biol 698: 167-184.

Rinaldi G (2000) In Flora dell'Arcipelago Toscano. Edizioni Archipelagos, Portoferraio (Livorno).

Ruffoni B, Pistelli L, Bertoli A, Pistelli L (2010) Plant Cell Cultures: Bioreactors for Industrial Production. Adv Exp Med Biol 698: 203221. 
Ruffoni B, Mascarello C (2009) Tecniche per la propagazione in vitro degli arbusti mediterranei. Flortecnica 6: 51-52.

Santos-Gomes PC, Fernandes-Ferreira M (2001) Organ- and season-dependent variation in the essential oil composition of Salvia officinalis L. cultivated at two different sites. I Agric Food Chem 49: 2908-2916. Scarpa GM, Milia M, Satta M 2000. The influence of growth regulators on proliferation and rooting of in vitro propagated myrtle Plant Cell Tiss Org Cult 62: 175-179.

Sefidkon F, Abbasi K, Khaniki GB (2006) Influence of drying and extraction methods on yield and chemical composition of the essential oil of Satureja hortensis. Food Chem 99: 19-23.
Stenhagen E, Abrahamsson S, McLafferty FW (1974) In Registry of mass spectral data. Wiley \& Sons, New York.

Swigar AA, Silverstein RM (1981) In Monoterpenes. Aldrich Chem. Comp., Milwaukee.

Zawirska-Wojtasiak R, Wasowicz E (2009) GC analysis of rosemary aroma isolated traditionally by distillation and by SPME. J Essent Oil Res 21: 8-15. 